\title{
State Space Reduction by Proving Confluence
}

\author{
Stefan Blom and Jaco van de Pol \\ CWI, P.O.-box 94.079, 1090 GB Amsterdam, The Netherlands \\ \{sccblom, vdpol\}@cwi.nl
}

\begin{abstract}
We present a modular method for on-the-fly state space reduction. The theoretical foundation of the method is a new confluence notion for labeled transition systems. The method works by adding confluence information to the symbolic representation of the state space. We present algorithms for on-the-fly exploration of the reduced state space, for detection of confluence properties and for a symbolic reduction, called prioritization. The latter two algorithms rely on an automated theorem prover to derive the necessary information. We also present some case studies in which tools that implement these algorithms were used.
\end{abstract}

Keywords: Labeled transition systems, on-the-fly state space reduction, partial order reduction, confluence, theorem proving, symbolic transformation, branching bisimulation, $\mu \mathrm{CRL}$ tool set

\section{Introduction}

A popular approach to the verification of distributed systems is based on an exhaustive state space exploration. This approach suffers from the well-known state space explosion problem. Much research is devoted to algorithms that generate a reduced, but essentially equivalent, state space. Collectively, these methods are called partial-order reduction methods. In this paper we introduce a new method for generating a reduced state space that is branching bisimilar with the original one.

The method is based on a subtle variation on the confluence-notion for labeled transition systems (LTS). Invisible $(\tau)$ steps in the LTS may be confluent or not. All states in a subset connected by confluent steps are branching bisimilar. By virtue of our new confluence notion, this subset may be replaced by a particular representative state, and only transitions outgoing from this representative need to be explored. This is explained in Section 2 .

In order to apply confluence for the immediate generation of the reduced state space, the confluent transitions must be detected before generating the LTS. This is solved in Section 3 by representing the system specification in an intermediate format, called linear process. A specification in this format consists of a finite number of symbolic transitions. The confluence property of each symbolic transition (or in fact a stronger approximation) can be expressed as a Boolean formula over the data types that occur in the specification. This formula is solved by a separate automated theorem prover. If the formula can be 
proved, the transition is marked as confluent, allowing for some reduction of the corresponding state space.

In some cases it is even possible to feed the information on confluent symbolic transitions back to the symbolic level. This results in a transformation on linear processes, which we call symbolic prioritization, described in Section 4. Confluence detection and symbolic prioritization can be applied to infinite state spaces as well. In Section 5 we show a number of applications, to which we applied our state space reduction techniques. The example in Section 5.2 goes beyond traditional partial-order reduction methods, which are based on super-determinism.

Implementation. Our ideas are implemented in the context of the $\mu \mathrm{CRL}$ tool set [3]. The basic functionality of this tool set is to generate a state space (LTS) out of a $\mu \mathrm{CRL}$ specification. To this end linear processes are used as an intermediate representation. This contributes to the modularity of the tool set. In particular, several optimizations are implemented as separate tools that transform a linear process, aiming at a reduction of the state space to be generated.

To this tool set, we added symbolic prioritization as yet another optimizer on linear processes. Moreover, the on-the-fly reduction algorithm has been integrated in the state space generator of the tool set.

With the approach in this paper we further contribute to modularity. In particular, we defined a notion of confluence, which is quite liberal, but nevertheless sufficient to ensure correctness of the on-the-fly reduction algorithm. Finding confluent transitions is a separate task. In fact, while the maximal set of confluent transitions is hard to detect, it is perfectly acceptable if actual confluence detection algorithms only find a subset.

We have used an automated theorem prover to find a reasonable subset of confluent transitions, but an alternative approach could be to prove confluence by hand, or with interactive theorem provers. In cases where the specification is automatically generated from source code, it is sometimes even possible to know a priori that certain transitions are confluent.

Related Work. Several partial order reduction algorithms that preserve branching bisimilarity have been proposed in the literature $[19,15,14,1]$. These approaches also allow the reduction to a representative subset of all states. Some of these approaches restrict attention to deterministic transitions. All these approaches involve some notion of determinacy. We compare our approach with [19], where criteria $\ddot{\mathrm{A}} 5$ and $\ddot{\mathrm{A}} 8$ for obtaining branching bisimilar reduced state spaces are introduced.

Criterion $\ddot{\mathrm{A}} 8$ allows the selection of one outgoing transition from a state, provided it is an invisible super-deterministic transition. In our approach, such a transition need not be deterministic, but only confluent, which means that eventually the computation paths reach the same state. It can be proved that the set of super-deterministic transitions forms a confluent set of $\tau$-transitions, but not vice versa. Criterion $\ddot{\mathrm{A}} 5$ prevents that a transition is postponed forever. This is implemented in [14] by the algorithm Twophase. In phase one, a state is 
expanded by repeatedly applying deterministic invisible transitions; the result is then fully expanded in phase two. Our algorithm can be seen as a modification: we take confluent transitions until a terminal strongly connected component (SCC) is reached, instead of deterministic transitions only.

Our method has some similarities with [1], where sequences of a component's local transitions are compressed to a single transition. Also, they have a detection phase and a generation phase. However, the setting is quite different because that paper aims at compositional model checking, whereas our method is independent of the structure of the specification. Moreover, they only prove preservation of weak-simulation equivalence.

Several confluence notions have been studied in the setting of branching bisimulation $[12,20,9]$. In [2] these notions are compared systematically. In summary, the notions in $[12,20]$ only deal with global confluence, whereas we deal with partial confluence, i.e. it suffices to identify a confluent subset of the $\tau$ transitions. In practical applications it is seldom the case that all $\tau$-transitions are confluent. The confluence notion in [20] has the nice theoretical property that the $\tau$-transition relation is confluent, if and only if it only connects states that are branching bisimilar. For reduction purposes, this would require to consider all outgoing transitions in each terminal SCC. Our notion of confluence is slightly stronger, and as a consequence we only have to take the outgoing transitions from a single member of each terminal SCC.

The confluence notion in [12] was adapted to partial confluence already in [9]. In order to apply it for state space reduction, this notion required the absence of $\tau$-loops. [9] introduced an algorithm to reduce concrete state spaces as follows. First, all strongly connected $\tau$-components are collapsed, in order to get rid of $\tau$-cycles. Then, the maximal set of strongly confluent $\tau$-steps is computed, and strongly confluent $\tau$-transitions are given priority over other transitions. We note that these steps can only be applied after generating the unreduced state space. Especially absence of $\tau$-loops is a severe obstacle for on-the-fly generation. In our paper, we use theorem proving to find (not necessarily all) confluent symbolic transitions in the specification. Our modified confluence notion works in the presence of $\tau$-loops. So we provide a genuine on-the-fly reduction method. Our new method even allows to perform some optimization at specification level already.

\section{Confluence and Reduction On-the-Fly}

In this section we present the confluence property, a state space reduction method based on the confluence property and an algorithm that computes these reduced state spaces "on-the-fly".

We use labeled transition systems (LTS) to model states spaces. Confluence is a property of sets of invisible transitions in an LTS. Any set of transitions induces an equivalence relation on the set of states, which identifies states in the same component. If a set of invisible transitions is confluent then the induced equivalence relation is a branching bisimulation. Moreover, each finite 
equivalence class has a representative, whose transitions are the same as those of the whole equivalence class. Because of these representatives, we can give an algorithm that computes the reduced state space without computing the whole original state space.

Because the reduction preserves branching bisimilarity, the reduced state space can be used to check all properties of the original system that are expressible in action based $\mathrm{CTL}^{*}-\mathrm{X}$ (computation tree logic without next-time) or HML (Hennessy-Milner logic).

\subsection{Confluence}

The labels of our LTSs will be taken from a given set Act. We assume that Act contains a special element $\tau$, representing the invisible action.

Definition 1 (LTS). A labeled transition system is a triple $\left(S, \rightarrow, s_{0}\right)$, consisting of a set of states $S$, transitions $\rightarrow \subseteq S \times$ Act $\times S$ and an initial state $s_{0} \in S$.

We write $s \stackrel{a}{\rightarrow} t$ for $(s, a, t) \in \rightarrow$. Moreover, $\stackrel{a}{\rightarrow}$ denotes the transitive reflexive closure of $\stackrel{a}{\longrightarrow}$, and $\stackrel{a}{\longleftrightarrow}$ denotes the equivalence relation induced by $\stackrel{a}{\longrightarrow}$, i.e. its reflexive, transitive, symmetric closure. We write $s \stackrel{a}{\Longrightarrow} t$ if either $s \stackrel{a}{\longrightarrow} t$, or $a=\tau$ and $s=t$. Note that in $s \stackrel{a}{\Longrightarrow} t$ only invisible steps are optional. Given a subset

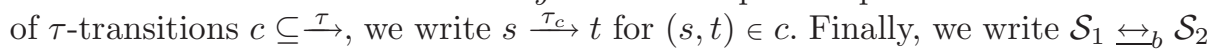
to denote that LTSs $\mathcal{S}_{1}$ and $\mathcal{S}_{2}$ are branching bisimilar [6].

The idea is that a subset $c$ of the set of invisible transitions is confluent if the steps in $c$ cannot make real choices. This is formalized with two conditions. First, if in a certain state two different sequences of $c$ steps are possible then these sequences can be extended with more $c$ steps to sequences that end in the same state. Second, if in a state both a $c$ step and an $a$ step are possible then after doing the $c$ step, the $a$ step is still possible (optional if $a=\tau$ ) and the results of the two $a$ steps are in the same $c$ equivalence class.

Definition 2 (confluence). Let $c$ be a subset of $\stackrel{\tau}{\longrightarrow}$. Then $c$ is confluent iff the following diagrams hold (for all $a \in$ Act):
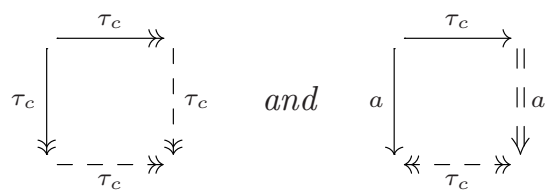

As a consequence, the equivalence relation $\stackrel{\tau_{c}}{\longleftrightarrow}$ induced by a confluent $c$ coincides with $\stackrel{\tau_{c}}{\longrightarrow}$. $\stackrel{\tau_{c}}{\longleftarrow}$. We can view $\stackrel{\tau_{c}}{\longrightarrow}$ as a directed graph. A strongly connected component (SCC) in this graph is a maximal set of states $X$, such that for all $s, t \in X$ we have $s \stackrel{\tau_{c}}{\longrightarrow} t$ and $t \stackrel{\tau_{c_{c}}}{\longrightarrow} s$. A terminal SCC (TSCC) is an SCC $X$ without outgoing edges, i.e. for all $s \in X$, if $s \stackrel{\tau_{c}}{\longrightarrow} t$ then $t \in X$. 


\subsection{Reduction}

As mentioned before, the equivalence relation induced by a confluent set is a branching bisimulation. By taking the original state space modulo this equivalence one can reduce the state space. An effective way of computing the transitions of the reduced state space is to find a representative of each equivalence class, whose transitions are precisely the transitions of the equivalence class. As representative of a class, we can choose any element in a TSCC of this class, if that exists. Note that in finite graphs every equivalence class contains a TSCC. Because of confluence this TSCC is unique. The notion of representation map is based on this idea. The first condition forces every element in an equivalence class to have the same representative. The second condition forces this representative to be in the TSCC.

Definition 3 (representation map). Given an $\operatorname{LTS} \mathcal{S} \equiv\left(S, \rightarrow, s_{0}\right)$ with a confluent subset of $\tau$-steps labeled $c$, a map $\phi: S \rightarrow S$ is called a representation map if $\forall s, t \in S: s \stackrel{\tau_{c}}{\longrightarrow} t \Rightarrow \phi(s)=\phi(t)$ and $\forall s \in S: s \stackrel{\tau_{c}}{\phi} \phi(s)$.

Based on the notion of representation map we can define a reduced LTS. The set of states of the reduced LTS will be the set of representatives. For every transition from a representative to a destination state in the original LTS, we include a transition from that representative to the representative of that destination in the reduced LTS. Finally, the new initial state is the representative of the old initial state. In [2] it is proven that the reduced LTS is branching bisimilar to the original LTS.

Definition 4 (LTS modulo $\phi$ ). Given a confluent $c \subseteq \stackrel{\tau}{\longrightarrow}$ and a representation map $\phi$, we define $\mathcal{S} / \phi=\left(\phi(S), \underset{\phi}{\longrightarrow}, \phi\left(s_{0}\right)\right)$, where $s \underset{\phi}{\vec{a}} t$ if $a \neq \tau_{c}$ and $\exists t^{\prime}: s \stackrel{a}{\longrightarrow} t^{\prime}$ and $\phi\left(t^{\prime}\right)=t$. As usual, $\phi(S)=\{\phi(s) \mid s \in S\}$.

Theorem 5 ([2]). Given a transition system $\mathcal{S}$ with a confluent subset of $\tau$-steps labeled $c$ and a representation map $\phi$, we have that $\mathcal{S} \biguplus_{b} \mathcal{S} / \phi$.

\subsection{Algorithm for Reduction On-the-Fly}

The essential functions in an "on-the-fly" interface for an LTS are a function that yields the initial state and a function that computes outgoing transitions of a given state. Given an on-the-fly representation of an LTS and the label for confluent transitions, the key to providing an on-the-fly representation of the reduced LTS is a function that computes a representation map (see Figure 1).

Such a function must return a representative from the terminal strongly connected component of the $\tau_{c}$ graph. Moreover, this representative must be unique for all elements of an equivalence class. We implemented the latter requirement simply by maintaining a table of pairs $(s$, Representative $(s))$ that we have computed so far. To compute a representative if it is not in the table, we use a variation on Tarjan's algorithm for finding strongly connected components [18]. 


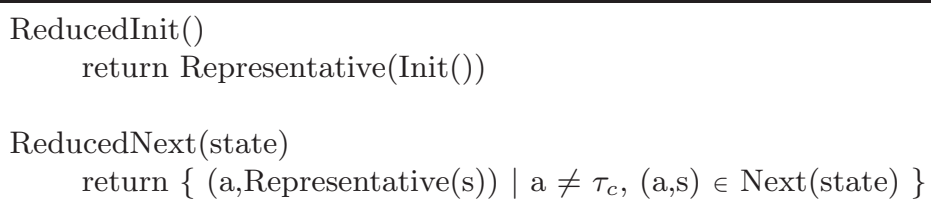

Fig. 1.

More precisely, we perform a depth first search of the graph of confluent transitions until we find a state with a known representative or until we backtrack from a node where we entered a strongly connected component. The first thus encountered component is the TSCC. In the latter case this node is chosen as the representative, and stored in the table (see [2] for a detailed algorithm).

The table consumes a significant amount of memory. If this is unacceptable, a total order on the set of states can be provided, and one can choose the least state in the TSCC as the representative and recompute the TSCC each time rather than storing it.

\section{Confluence Detection by Theorem Proving}

In the previous section we discussed a state space generation algorithm, which applies reduction on-the-fly, based on some information on confluent transitions. In this section we show how this information is obtained. In particular, by exploiting a special format for the specification, we show that (stronger approximations of) confluence properties can be expressed by quantifier-free first order formulae. These formulae can be solved by a separate automated theorem prover.

\subsection{Symbolic Specifications in $\mu$ CRL}

We implemented our ideas in the setting of the $\mu \mathrm{CRL}$ formalism. A $\mu \mathrm{CRL}$ specification [11,3] consists of an algebraic specification, defining the data sorts, and a process specification, defining the system under scrutiny as the parallel composition of several components, each specified as a process algebra expression. We utilize the result in [10], that such specifications can be transformed to a linear process, without a considerable blow-up in size.

A linear process consists of a vector of global state variables $d$, an initial state vector $d_{0}$, and a set of program rules, traditionally called summands. These summands define the process behaviour in a condition/action/effect style, similar to I/O-automata or UNITY programs. These summands have the following form ${ }^{1}$ :

$$
\left\{\sum_{e_{i}}\left[b_{i}\left(d, e_{i}\right)\right] \Rightarrow a_{i}\left(d, e_{i}\right) ; d:=g_{i}\left(d, e_{i}\right)\right\}_{i \in I}
$$

\footnotetext{
${ }^{1}$ We focus on the essential ingredients, rather than concrete $\mu$ CRL syntax.
} 
We assume that the data algebra contains the special sorts Bool for booleans and Act for external actions. In the summands above, we have that:

$-e_{i}$ is a vector of local variables, used for instance to model arbitrary input of this summand.

$-b_{i}\left(d, e_{i}\right)$ is a term of type Bool, with variables among $d$ and $e_{i}$, denoting the condition or guard of the summand.

- $a_{i}\left(d, e_{i}\right)$ is a term of type Act, with variables among $d$ and $e_{i}$, denoting the action executed by this summand.

- $g_{i}\left(d, e_{i}\right)$ is a vector of terms, whose sorts match the vector of global state variables. These denote the next state.

Each summand specifies a structural transition $\rightarrow \vec{i}$ as follows:

$$
d \underset{i}{\stackrel{\alpha}{l}} d^{\prime} \text { iff } \exists e_{i} . b_{i}\left(d, e_{i}\right) \wedge d^{\prime}=g_{i}\left(d, e_{i}\right) \wedge \alpha=a_{i}\left(d, e_{i}\right)
$$

Together, a linear process specifies a structural labeled transition system $\left(\Sigma,(\vec{i})_{i \in I}, s_{0}\right)$, from which the ordinary LTS can be obtained by taking the union of all structural transitions. Here a state in $\Sigma$ is a vector of data values for the global variables; $s_{0}$ is the initial state vector; $I$ is the (index) set of the summands; $\vec{i}$ is the structural transition generated by summand $i$, which is a subset of the transitions of the whole LTS.

Note that a structural transition defined in this way is partial (due to the enabling condition $b$ ) and non-deterministic (due to choice involved in $\exists e$ ). Also note that one summand may generate transitions with various labels. A similar decoupling of action labels and structural transitions occurs in [19].

\subsection{Generation of Confluence Formulae}

Owing to the format of linear processes, commutation formulae can be generated. In order to facilitate automated theorem proving, we try to avoid quantifiers. The generated formulae will be Boolean expressions over the user defined abstract data types, with implicit universal quantification.

To get the formulae in this form, we only consider a special case, which occurs frequently in practice. So in fact we detect a stronger approximation of confluence. Consider two divergent steps of summands $i$ and $j$ :

$$
\begin{gathered}
\sum_{e_{i}}\left[b_{i}\left(d, e_{i}\right)\right] \Rightarrow a_{i}\left(d, e_{i}\right) ; d:=g_{i}\left(d, e_{i}\right) \\
\sum_{e_{j}}\left[b_{j}\left(d, e_{j}\right)\right] \Rightarrow \tau ; d:=g_{j}\left(d, e_{j}\right)
\end{gathered}
$$

The first simplification is that we only consider a closing of this diagram in zero or one step (strong confluence). Furthermore, we assume that the diagram is closed by using the same summands $j$ and $i$ again, and moreover we only try 
the same instance of summand $i$ and $j$. This situation is depicted in the following picture (we left out the enabling conditions).
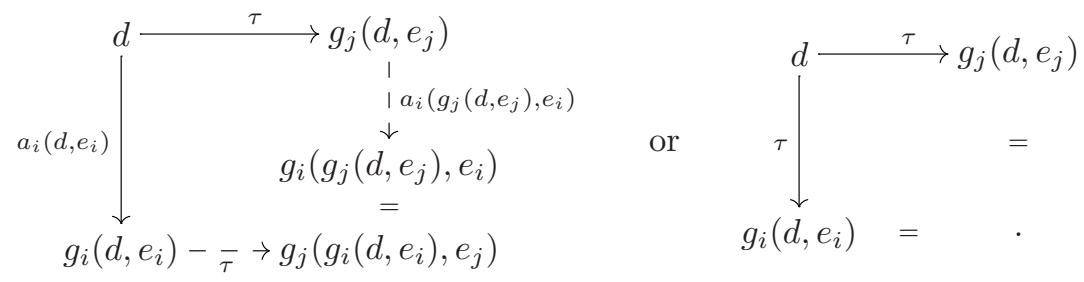

Commutation of $\tau$-summand $j$ with summand $i$ can be expressed by the following Boolean expression over the algebraic data theory. Note that these formulae can be generated automatically for any linear process.

$$
\left(\begin{array}{c}
b_{i}\left(d, e_{i}\right) \\
\wedge \\
b_{j}\left(d, e_{j}\right)
\end{array}\right) \rightarrow\left(\begin{array}{ll}
b_{i}\left(g_{j}\left(d, e_{j}\right), e_{i}\right) & \hat{y} \\
b_{j}\left(g_{i}\left(d, e_{i}\right), e_{j}\right) & \wedge \\
a_{i}\left(d, e_{i}\right)=a_{i}\left(g_{j}\left(d, e_{j}\right), e_{i}\right) & \wedge \\
g_{i}\left(g_{j}\left(d, e_{j}\right), e_{i}\right)=g_{j}\left(g_{i}\left(d, e_{i}\right), e_{j}\right)
\end{array}\right) \vee\left(\begin{array}{c}
a_{i}\left(d, e_{i}\right)=\tau \\
\wedge \\
g_{i}\left(d, e_{i}\right)=g_{j}\left(d, e_{j}\right)
\end{array}\right)
$$

If $\tau$-summand $j$ commutes with all summands $i$ (including $j$ ), it can be safely marked as a confluent $\tau$-summand. As strong confluence implies confluence, the transitions generated by $\tau$-summand $j$ will form a confluent subset in the sense of Definition 2. Because the union of two confluent subsets constitutes a confluent subset, it is safe to label multiple summands in the same linear process.

\subsection{Automated Theorem Prover}

In order to prove formulae of the above kind, we have built a theorem prover for Boolean combinations over a user-defined algebraic data type. In [17] we show how an extension of binary decision diagrams (BDD) enhanced with term rewriting can be applied to these formulae. This is along the lines of the BDDs extended with equality developed in [8]. Given a formula, the prover returns an equivalent but ordered BDD. If this BDD equals TRUE, the pair $(i, j)$ commutes.

If the resulting BDD doesn't equal TRUE, then the formula could not be proved, and $\tau$-summand $j$ cannot be marked as confluent. Note that this may be due to the fact that it is not confluent, or due to the fact that the prover is inherently incomplete (simple equalities over an abstract data type are undecidable already, let alone arbitrary Boolean expressions). In this case, the prover provides some diagnostics, on the basis of which user interaction is possible.

The user can add equations to the data specification, or provide an invariant. It is possible to add new equations, provided they hold in the initial model. Proving correctness of the new equations requires induction, which is beyond our theorem prover. The new equations could be proved either manually, or using a separate interactive theorem prover.

In some cases, the formula is not valid in the initial model, but it would hold for reachable states $d$. In this case, the user may supply an invariant Inv and the 
confluence formulae are proved under the assumption Inv $(d)$. Of course such an invariant must be checked separately. With our theorem prover, one can check whether Inv holds initially, and is preserved by all summands $i$ :

$$
\operatorname{Inv}\left(d_{0}\right) \text { and for all } i \in I: b_{i}\left(d, e_{i}\right) \wedge \operatorname{Inv}(d) \rightarrow \operatorname{Inv}\left(g_{i}\left(d, e_{i}\right)\right)
$$

\section{Optimization by Symbolic Prioritization}

Combining the previous sections, we can now mark certain transitions as being confluent by using an automated theorem prover, and subsequently generate a reduced state space by the on-the-fly reduction algorithm. However, the confluence marks can also be used to apply an optimization to the specification, i.e. on the symbolic level. Let transition $j$ be a deterministic transition (i.e. without local variables) which is marked as confluent:

$$
\left[b_{j}(d)\right] \Rightarrow \tau_{c} ; d:=g_{j}(d)
$$

Now because the $j$ summand is confluent, it may be given priority to other summands, as long as loops are avoided. To avoid loops, we will only give summand $j$ priority just after a non-marked (visible or non-visible) transition. So let another summand $i$ be given, which is not marked as confluent:

$$
\sum_{e_{i}}\left[b_{i}\left(d, e_{i}\right)\right] \Rightarrow a_{i}\left(d, e_{i}\right) ; d:=g_{i}\left(d, e_{i}\right)
$$

Now, if we can prove that $j$ is always enabled after the $i$-transition, we can combine the $i$ and $j$ summand in one step. Enabledness of $j$ can be represented by the formula $b_{i}\left(d, e_{i}\right) \rightarrow b_{j}\left(g_{i}\left(d, e_{i}\right)\right)$. This formula is sent to the prover, and upon success, we modify summand $i$ to become:

$$
\sum_{e_{i}}\left[b_{i}\left(d, e_{i}\right)\right] \Rightarrow a_{i}\left(d, e_{i}\right) ; d:=g_{j}\left(g_{i}\left(d, e_{i}\right)\right)
$$

We call this transformation symbolic prioritization. One advantage of this symbolic optimization is that the intermediate state $g_{i}\left(d, e_{i}\right)$ doesn't have to be explored during state space generation. Another advantage is that this optimization often gives rise to a cascade of other possible optimizations, such as removal of dead code and unused variables. For instance, summand $j$ might be unreachable after the optimization. This can be checked by proving that $\neg b_{j}$ is an invariant. Other summands may become unreachable as well, and variables used in them may become useless and can be removed or given a dummy value.

A very interesting effect is that we can now possibly mark more transitions as confluent. Recall that we only mark "strong confluence", where a diverging pair is closed in zero or one step. After symbolic prioritization, we might detect confluence also when the diverging pair can be closed in two steps, as illustrated in the diagram below. Of course, this process can be iterated. 


\begin{tabular}{|l||r|r||r|r||r|r|}
\hline \multicolumn{1}{|c||}{} & original state space & reduced state space & \multicolumn{2}{c|}{ total costs } \\
\hline system & states & transitions & states & transitions & states & transitions \\
\hline \hline abp & 97 & 122 & 29 & 54 & 97 & 98 \\
\hline brp & 1952 & 2387 & 1420 & 1855 & 1952 & 2275 \\
\hline mutex & 96 & 192 & 26 & 46 & 56 & 75 \\
\hline DKR(3) & 67 & 124 & 2 & 1 & 20 & 19 \\
\hline DKR(5) & 864 & 2687 & 2 & 1 & 32 & 31 \\
\hline DKR(7) & 18254 & 77055 & 2 & 1 & 72 & 71 \\
\hline Firewire(10) & 72020 & 389460 & 6171 & 22668 & 8443 & 23326 \\
\hline Firewire(12) & 446648 & 2853960 & 27219 & 123888 & 40919 & 127016 \\
\hline Firewire(14) & 2416632 & 17605592 & 105122 & 544483 & 167609 & 557419 \\
\hline Lift1 & 38000 & 112937 & 12826 & 38151 & 27292 & 48684 \\
\hline Lift2 & 223720 & 712593 & 69987 & 231365 & 166645 & 300051 \\
\hline Lift1 + prio & 38000 & 112937 & 12826 & 38151 & 16404 & 39991 \\
\hline Lift2 + prio & 223720 & 712593 & 69987 & 231365 & 88741 & 239958 \\
\hline
\end{tabular}

Fig. 2. Benchmarks for confluence detection and on-the-fly reduction

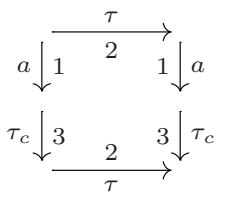

becomes after prioritization

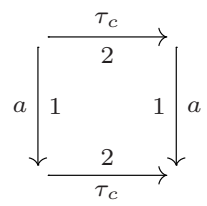

In the system on the left, automatic confluence marking will not detect the confluence of $\tau$-summand (2), because the divergence with $a$-summand (1) cannot be closed in one step. However, typically $\tau$-summand (3) will be detected to be confluent, because no other summands are enabled in its source state. The marking of summand (3) is denoted by the $\tau_{c}$-label. Note that after $a$-summand (1) it is always possible to perform the marked $\tau_{c}$-summand (3). Hence symbolic prioritization can be applied, and we obtain the system on the right. In the new situation summand (2) becomes strongly confluent, so it will be detected by a second application of automatic confluence detection. Due to the confluence of summand (2), the state space generation algorithm will now visit a single path through this graph. Also note that summand (3) becomes unreachable in this example.

\section{Applications}

We applied our method to finite instances of several distributed algorithms, protocols and industrial case studies. A number of experiments are described in detail in [17]. The $\mu$ CRL-code is available via http://www.cwi.nl/ ${ }^{\sim}$ dpol/ 
CAV02-experiments. Figure 2 shows the reduction obtained by confluence detection and on-the-fly reduction. For each system, we list the size of the original and the reduced state space, and also - in order to allow fair comparisons - the total costs including the number of nodes and transitions that are visited during the TSCC-computation.

The first rows refer to the alternating bit protocol, the bounded retransmission protocol, and a mutual exclusion algorithm. Furthermore, $\operatorname{DKR}(n)$ refers to the DKR leader election protocol with $n$ parties; Firewire $(n)$ to the Firewire Tree Identify protocol for $n$ components from the IEEE 1394 bus standard; and the lift entries refer to a case study with distributed lifts [7], used for lifting car trucks by several lift legs. For the lift systems we also denote the cost reduction obtained after symbolic prioritization.

As a conclusion, we note that the contribution of confluence reduction to toy examples is rather modest. However, on the industrial case studies (Firewire, Lift) the reduction is notable. On the DKR protocol the reduction is even dramatic (the number of visited states goes from exponential down to $n \log n$ ). We now discuss two experiments in more detail.

\subsection{Leader Election Protocol}

In the DKR (Dolev-Klawe-Rodeh) leader election protocol [5], $n$ parties are connected in a ring by $n$ channels, modeled as unbounded queues. These parties exchange messages, which are not visible for the outside world. After a finite number of messages, the party with the highest identification performs the action "I'm the leader".

This algorithm allows for a remarkable state space reduction, viz. from exponential to linear in the number of parties. The theorem prover detects that all $\tau$-summands are confluent, even when $n$ is unknown. Given a concrete number of parties, the generation algorithm finds a completely deterministic path representing the whole state space. So the state space is immediately reduced to a single transition, labeled "I'm the leader". We remark that also the traditional partial order reduction methods have been successfully applied to this example (see also [1]).

\subsection{Shared Data Space Systems}

We also studied distributed systems based on shared data space architectures, such as Splice [4]. A Splice system consists of a number of application processes, that coordinate through agents, which are coupled via some network. The agents locally maintain multi-sets of data items (the distributed data space), into which applications can write new items, and from which applications can read items. The agents distribute their items by asynchronously sending messages to each other over the network.

Figure 3 depicts a simple Splice system, with a producer and a consumer. In between, several workers independently take input items from their local storage, perform some computation, and write output items back in the space. We want 


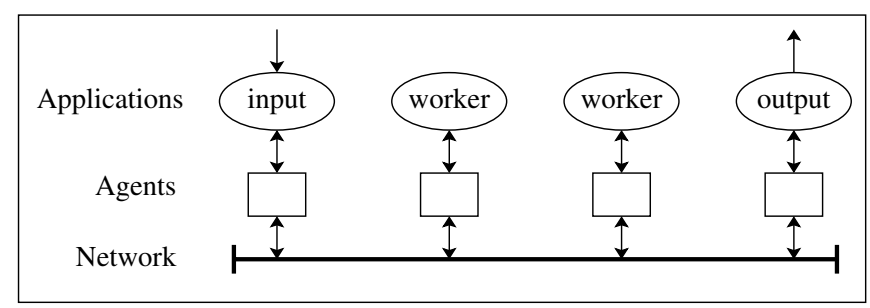

Fig. 3. Splice architecture

\begin{tabular}{|l||r||r|r||r|r|}
\hline \multicolumn{1}{|c||}{} & \multicolumn{1}{|c||}{ original space } & reduced state space & after prioritization \\
\hline & & \# states & cost & \# states & cost \\
\hline \hline Splice(1,2) & 85362 & 15 & 75 & 9 & 45 \\
\hline Splice(2,2) & 18140058 & 69 & 644 & 9 & 65 \\
\hline Splice(3,2) & $? ?$ & 297 & 5151 & 9 & 101 \\
\hline Splice(1,4) & $? ?$ & 83 & 743 & 25 & 169 \\
\hline Splice(2,4) & $? ?$ & 1661 & 29936 & 25 & 249 \\
\hline Splice(3,4) & $? ?$ & 31001 & 1057187 & 25 & 393 \\
\hline Splice(1,6) & $? ?$ & 317 & 3657 & 56 & 425 \\
\hline Splice(2,6) & $? ?$ & 14387 & 326832 & 56 & 630 \\
\hline Splice(3,6) & $? ?$ & $? ?$ & $? ?$ & 56 & 999 \\
\hline
\end{tabular}

Fig. 4. Splice benchmarks with symbolic prioritization

to prove transparency of the number of workers. See [13] for the full case study, which heavily relies on using our confluence reduction.

This communication mechanism is a-synchronous, and leads to much nondeterminism: messages from one agent are sent to the others in any order. Consequently, the agents receive messages in various orders, even when they originate from the same agent. By proving confluence, it is detected that all these different orders are equivalent. In fact, the on-the-fly reduction algorithm computes a reduced state space, as if there were only one global multi-set of data items.

Another reduction is possible within the workers. They read any message from their agent, and write some computed result back. Note that such transactions cannot be represented by super-deterministic transitions, because a worker can start with any message in the current set of its agent. Therefore, traditional partial-order reduction methods, which are based on super-determinism, fail on this example. However, several such transactions commute, basically because $(A \cup\{a\}) \cup\{b\}=(A \cup\{b\}) \cup\{a\}$. Using confluence reduction, only a fixed transaction order is explored.

For this example, we also needed symbolic prioritization: although the transactions commute, the corresponding diagrams can only be closed in multiple steps. This corresponds to the diagram in Section 4. 
In Figure 4 we applied our reductions on the $\operatorname{Splice}(m, n)$ benchmarks, having $m$ workers and processing $n$ input values. We show the number of generated states, as well as the total number of visited states, including those used in the TSCC search. The size of the original state space (first column) could only be computed in a few cases, and even here we used a parallel machine. After one application of confluence detection and on-the-fly reduction, in most cases the state space could be generated, but this approach doesn't scale well (middle two columns). After symbolic prioritization more transitions could be proven confluent, and running on-the-fly reduction again results in pretty small state spaces (last two columns).

As a final remark, we note that the size of the reduced space doesn't depend on the number of workers anymore. So this example has been solved nearly symbolically in the number of workers.

\section{References}

1. R. Alur and B.-Y. Wang. "Next" heuristic for on-the-fly model checking. In J. C. M. Baeten and S. Mauw, editors, Proc. of CONCUR '99, LNCS 1664, pages 98-113. Springer, 1999. 597, 598, 606

2. S. C. C. Blom. Partial $\tau$-confluence for efficient state space generation. Technical Report SEN-R0123, CWI, Amsterdam, 2001. 598, 600, 601

3. S. C. C. Blom, W. J. Fokkink, J. F. Groote, I. van Langevelde, B. Lisser, and J. C. van de Pol. $\mu$ CRL: A toolset for analysing algebraic specifications. In G. Berry, etal, editor, Proc. of CAV 2001, LNCS 2102, pages 250-254. Springer, July 2001. 597, 601

4. M. Boasson. Control systems software. IEEE Transactions on Automatic Control, 38(7):1094-1106, July 1993. 606

5. D. Dolev, M. Klawe, and M. Rodeh. An $O(n \log n)$ unidirectional distributed algorithm for extrema finding in a circle. Journal of Algorithms, 3(3):245-260, September 1982. 606

6. R. J. van Glabbeek and W. P. Weijland. Branching time and abstraction in bisimulation semantics. Journal of the ACM, 43(3):555-600, 1996. 599

7. J. F. Groote, J. Pang, and A. G. Wouters. A balancing act: Analyzing a distributed lift system. In S. Gnesi and U. Ultes-Nitsche, editors, Proc. of FMICS, pages 1-12, Paris, France, 2001. 606

8. J. F. Groote and J. C. van de Pol. Equational binary decision diagrams. In M. Parigot and A. Voronkov, editors, Proc. of LPAR 2000, LNAI 1955, pages 161-178. Springer, 2000. 603

9. J. F. Groote and J. C. van de Pol. State space reduction using partial $\tau$-confluence. In M. Nielsen and B. Rovan, editors, Proc. of MFCS 2000, LNCS 1893, pages 383393. Springer, 2000. 598

10. J. F. Groote, A. Ponse, and Y. S. Usenko. Linearization in parallel pCRL. Journal of Logic and Algebraic Programming, 48(1-2):39-70, 2001. 601

11. J. F. Groote and M. A. Reniers. Algebraic process verification. In J. A. Bergstra, A. Ponse, and S. A. Smolka, editors, Handbook of Process Algebra, chapter 17. Elsevier, 2001. 601

12. J. F. Groote and M. P. A. Sellink. Confluence for process verification. Theoretical Computer Science, 170:47-81, 1996. 598 
13. J. M. M. Hooman and J. C. van de Pol. Formal verification of replication on a distributed data space architecture. In Proceedings of SAC 2002 (Madrid), pages 351-358. ACM, 2002. 607

14. R. Nalumasu and G. Gopalakrishnan. An efficient partial order reduction algorithm with an alternative proviso implementation. Formal Methods in System Design, 20(3):231-247, 2002. 597

15. D. Peled. Partial order reduction: Linear and branching temporal logics and process algebras. In Peled et al. [16], pages 233-258. 597

16. D. A. Peled, V. R. Pratt, and G. J. Holzmann, editors. Partial Order Methods in Verification, DIMACS Series 29. AMS, July 1997. 609

17. J. C. van de Pol. A prover for the $\mu$ CRL toolset with applications - Version 0.1. Technical Report SEN-R0106, CWI, Amsterdam, 2001. 603, 605

18. R. E. Tarjan. Depth first search and linear graph algorithms. SIAM Journal on Computing, 1(2):146-160, 1972. 600

19. A. Valmari. Stubborn set methods for process algebras. In Peled et al. [16], pages 213-232. 597, 602

20. M. Ying. Weak confluence and $\tau$-inertness. Theoretical Computer Science, 238:465475, 2000. 598 